\title{
Rethinking the need for overnight admission after robotic-assisted laparoscopic prostatectomy
}

\author{
Srinath Kotamarti ${ }^{1} \cdot$ Thomas Williams $^{2} \cdot$ Michael Silver $^{1,3} \cdot$ David A. Silver $^{1} \cdot$ Ariel A. Schulman $^{1}$
}

Received: 11 June 2020 / Accepted: 24 June 2020 / Published online: 29 June 2020

๑) Springer-Verlag London Ltd., part of Springer Nature 2020

\begin{abstract}
Robotic-assisted laparoscopic prostatectomy (RALP) is the gold standard for the surgical management of localized prostate cancer (PCa). Multi-institutional series have demonstrated complications and readmissions in less than 5\% of patients and most are now discharged within $24 \mathrm{~h}$ of surgery. Recently, several high-volume surgeons demonstrated the safety of sameday discharge (SDD) after RALP. The main benefits include lower costs and reduced exposure to nosocomial infections and hospital errors. The leading arguments for criticism include potential suboptimal postoperative care and the risk of missing a catastrophic event. In recent years, important advances have further strengthened the argument for SDD including more structured perioperative care, integration of single-port robotic systems, and new challenges presented by the coronavirus 2019 (COVID-19) pandemic. Here, we provide further evidence demonstrating the safety of SDD in a multi-institutional cohort of patients and review the main arguments supporting the expanded use of this approach.
\end{abstract}

Keywords Prostate cancer $\cdot$ Radical prostatectomy $\cdot$ Robotic surgery $\cdot$ Same-day discharge

\section{Introduction}

Robotic-assisted laparoscopic prostatectomy (RALP) is the gold standard for the surgical management of localized prostate cancer $(\mathrm{PCa})$. Compared to the traditional open approach, RALP has demonstrated fewer complications, less blood loss, and decreased length of stay (LOS) [1]. Contemporary rates of complications and readmissions after RALP

Ariel A. Schulman

Aschulman@maimonidesmed.org

Srinath Kotamarti

Srikotamarti@gmail.com

Thomas Williams

Twilliams21@northwell.edu

Michael Silver

Michael.silver@downstate.edu

David A. Silver

Dsilver@maimonidesmed.org

1 Maimonides Medical Center, 745 64th St. 4th Floor, Brooklyn, NY 11220, USA

2 Hofstra-Northwell School of Medicine, 450 Lakeville Rd, Suite M42, Lake Success, NY 11042, USA

3450 Clarkson Ave, BSB 4-32, Brooklyn, NY 11203, USA occur in less than $5 \%$ and $4 \%$ of patients, respectively [2]. Advances in regional anesthesia including the use transversus abdominis plane and quadratus lumborum blocks have further reduced postoperative pain, eliminating the need for parenteral medications in most patients [3]. LOS after RALP at most centers is now 1-2 days [2].

In recent years, several high-volume surgeons have reported the safety of same-day discharge (SDD) after RALP $[4,5]$. There are several arguments supporting this practice. First, SDD offers a significant cost savings to the healthcare system. In a series of 500 men offered SDD, Abaza et al. calculated a yearly cost savings over $\$ 345,000$, with no major complications and only a $1.6 \%$ rate of readmission [4]. Second, avoiding hospitalization may expedite the recovery process. Bajpai et al. found that patients discharged the day of surgery had less pain, less interference with general activities and better-perceived overall health than those who stayed overnight [5]. Third, SDD decreases the risk of nosocomial infections and hospital error. Health care-associated infections occur in 3-4\% of hospitalized patients and significant medical error may occur in more than 250,000 hospitalized patients annually [6, 7].

There remain significant concerns about the widespread implementation of SDD. Most importantly, overnight observation provides a period for close monitoring to identify early 
complications, optimize pain control, and ensure that patients are on the path to recovery. Recent studies utilizing both subjective patient surveys and the Post-Anesthesia Discharge Scoring System (PADSS) highlighted potential barriers to SDD after RALP $[8,9]$.

But, the practice of overnight hospitalization may be a function of surgeon preference and historical custom rather than evidence-based practice. To our knowledge, there have been no reports of early catastrophic events immediately after discharge and no data showing a correlation between early discharge and increased emergency room visits or readmissions. Current studies questioning the feasibility of SDD have examined single-surgeon or single-center series that may be unfairly influenced by individual or local practice patterns.

To further evaluate safety, we examined SDD after RALP in a contemporary cohort of men recorded in the American College of Surgeons National Surgical Quality Improvement Program (NSQIP) database. From 2015 to 2017, we identified 157 patients who were discharged the day of surgery $(\operatorname{LOS}=0)$. SDD cases were propensity score matched based on age, body mass index (BMI), American Society of Anesthesia (ASA) score, and race in a 1:3 ratio with 458 men who underwent standard-length discharge (SLD) (LOS $=1-2$ days). Average age for both groups was 60 years. Caucasian ethnicity was identified in $72.3 \%$ and $72.9 \%$ of SDD and SLD groups, respectively. All matching parameters were similar between the groups $(p>0.05)$. Operation time was different, with SDD patients on average having shorter times (184.6 vs. 213.0, $p<0.0001$ ).

We then compared postoperative outcomes between the matched SDD and SLD groups. Minor Clavien-Dindo complications (1 and 2) were 1.94\% and $2.84 \%$ between SDD and SLD groups, respectively, while major Clavien-Dindo complications (3 and 4) were $1.29 \%$ and $1.31 \%$, respectively. The observed differences were not statistically significant. Similarly, no significant differences were found between groups with regard to discharge home, reoperation within 30 days, and readmission within 30 days (Table 1).

The matched-pairs analysis reinforces the non-inferior perioperative outcomes of SDD after RALP. These findings deserve attention because of the established accuracy of NSQIP complications recording methodology and the inclusion of both large and small hospitals across the country. To our knowledge, this is the first multi-institutional data demonstrating the safety of SDD. The main limitations include the retrospective nature, absence of pathologic correlation, and lack of data on surgeon volume and experience.
Table 1 Analysis of outcomes between the matched groups

\begin{tabular}{llll}
\hline & $\begin{array}{l}\text { Same day } \\
N=155\end{array}$ & $\begin{array}{l}1-2 \text { days } \\
N=458\end{array}$ & $p$ value \\
\hline $\begin{array}{llll}\text { Operation time (min) } \\
\text { Clavien-Dindo complication }\end{array}$ & $184.6(85.9)$ & $213.0(77.8)$ & 0.0001 \\
0 & $150(96.77 \%)$ & $439(95.85 \%)$ & \\
1 & $0(0 \%)$ & $2(0.44 \%)$ & \\
2 & $3(1.94 \%)$ & $11(2.4 \%)$ & \\
3 & $0(0 \%)$ & $1(0.22 \%)$ & \\
4 & $2(1.29 \%)$ & $5(1.09 \%)$ & \\
Discharged home & $152(98.1 \%)$ & $455(99.3 \%)$ & 0.173 \\
reoperation & $2(1.3 \%)$ & $4(0.9 \%)$ & 0.6458 \\
Readmission (30 days) & $6(3.9 \%)$ & $15(3.3 \%)$ & 0.7984 \\
\hline
\end{tabular}

\section{New challenges and new strategies}

Beyond accruing data on feasibility, new challenges presented by the coronavirus 2019 (COVID-19) pandemic and new technological innovations further justify the widening application of SDD after RALP. The COVID-19 pandemic has introduced a novel infectious risk to both patients and healthcare providers. A night in the hospital after surgery exposes patients to additional floors, personnel and other patients increasing the risk of contracting the virus. Further, economic ramifications of the pandemic have created increasing pressures to deliver the most cost-effective surgical care. Avoiding an overnight stay can save over $\$ 2000$ per case [4].

Implementation of more structured pre- and postoperative teaching and home monitoring will further enhance the success of SDD. Ploussard et al. demonstrated that structured prehabilitation and enhanced recovery after surgery (ERAS) pathways progressively increased the number of patients safely discharged the day of surgery after RALP with lower cost and similar complications [10]. Home cardiovascular monitoring technologies and virtual communication systems are evolving rapidly and will further expedite the transition to SDD. Remote-automated monitoring systems designed to follow patients at home after surgery are already in the advanced testing and implementation phases.

The adoption of SDD will be further enhanced by new surgical systems and techniques. The da Vinci SP ${ }^{\circledR}$ (Intuitive Surgical, Sunnyvale, CA, USA) single-port robotic system reduces skin incisions and tissue manipulation with the potential for decreased postoperative pain and earlier convalescence [11]. The system also facilitates extraperitoneal radical prostatectomy with demonstrated shorter postoperative stays and decreased postoperative narcotics, compared to the traditional transperitoneal approach [12]. 
The commercial expansion of the single-port system in the coming years is likely to further expand SDD.

In the near future, the transition to SDD after RALP will evolve due to the continued drive for cost-effective surgical care, pressures to reduce hospital contact during the COVID-19 pandemic and rapid advances in surgical technologies, home monitoring, and virtual communication platforms. While more robust evidence supporting SDD after RALP is accruing, we must be willing to reconsider previous surgical practices in the context of a rapidly changing healthcare environment.

Funding All authors have read and approved the manuscript, which is not under consideration elsewhere. No specific funding was allocated to this work.

\section{Compliance with ethical standards}

Conflict of interest All authors declare that they have no conflicts of interest. No author has direct or indirect commercial financial incentive associated with publishing the article.

Human and animal rights statement This article does not contain any studies with human or animal subjects performed by any of the authors.

\section{References}

1. Kowalczyk KJ, Levy JM, Caplan CF et al (2012) Temporal national trends of minimally invasive and retropubic radical prostatectomy outcomes from 2003 to 2007: results from the 100\% Medicare sample. Eur Urol 61:803-809

2. Xia L, Taylor BL, Pulido JE et al (2017) Predischarge predictors of readmissions and postdischarge complications in robot-assisted radical prostatectomy. J Endourol 31(9):864-871

3. Dudderidge TJ, Doyle P, Mayer EK, Taylor J, Agrawal S, Stolzenburg JU, Winkler MH (2012) Evolution of care pathway for laparoscopic radical prostatectomy. J Endourol. 26(6):660-665
4. Abaza R, Martinez O, Ferroni MC, Bsatee A, Gerhard RS (2019) Same day discharge after robotic radical prostatectomy. J Urol 202(5):959-963

5. Bajpai RR, Razdan S, Barack J, Sanchez MA, Razdan S (2019) Ambulatory robot-assisted laparoscopic prostatectomy: is it ready for prime time? A quality of life analysis. J Endourol 33(10):814-822

6. Magill SS, O'Leary E, Janelle SJ et al (2018) Changes in prevalence of health care-associated infections in US hospitals. N Engl J Med 379(18):1732-1744. https://doi.org/10.1056/NEJMoa1801 550

7. Makary MA, Daniel M (2016) Medical error-the third leading cause of death in the US. BMJ 353:i2139

8. Congnard D, Vincendeau S, Lahjaouzi A et al (2019) Outpatient robot-assisted radical prostatectomy: a feasibility study. Urology 128:16-22

9. Dobbs RW, Nguyen TT, Shahait M et al (2020) Outpatient robotassisted radical prostatectomy: are patients ready for same-day discharge? J Endourol. 34(4):450-455

10. Ploussard G, Almeras C, Beauval JB, Gautier JR, Loison G, Salin A, Tollon C (2020) Same-day discharge surgery for robot-assisted radical prostatectomy in the era of ERAS and prehabilitation pathways: a contemporary, comparative, feasibility study. World J Urol. https://doi.org/10.1007/s00345-020-03119-w

11. Aminsharifi A, Sawczyn G, Wilson CA, Garisto J, Kaouk J (2020) Technical advancements in robotic prostatectomy: singleport extraperitoneal robotic-assisted radical prostatectomy and single-port transperineal robotic-assisted radical prostatectomy. Transl Androl Urol 9(2):848-855. https://doi.org/10.21037/ tau.2019.11.35

12. Kaouk J, Aminsharifi A, Wilson CA et al (2020) Extraperitoneal versus transperitoneal single port robotic radical prostatectomy: a comparative analysis of perioperative outcomes. J Urol 203(6):1135-1140

Publisher's Note Springer Nature remains neutral with regard to jurisdictional claims in published maps and institutional affiliations. 